\title{
SELECTION OF TECHNICAL RISK RESPONSES FOR EFFICIENT CONTINGENCIES
}

Edouard Kujawski, Ph.D.

Systems Engineering Department

Engineering Division

Lawrence Berkeley National Laboratory

MS 46A-1123

Berkeley, California 94720

E-mail:_e_kujawski@lbl.gov

Tel: $510-486-6932$

Fax: 510-486-4711

February 2002

\begin{abstract}
The primary goal of good project risk management should be to successfully deliver projects for the lowest cost at an acceptable level of risk. This requires the systematic development and implementation of a set of Risk Response Actions (RRA) that achieves the lowest total project cost for a given probability of success while meeting technical performance and schedule. We refer to this set as the "efficient RRA set". This work presents a practical and mathematically sound approach for determining the efficient RRA set. It builds on some of Markowitz' s portfolio selection principles and introduces several conceptual and modeling differences to properly treat project technical risks. The set of RRAs is treated as whole and not just individual risks. The efficient RRA set is determined based on "Outcome Cost Vs Probability of Success". The risks and RRAs are characterized using scenarios, decision trees, and cumulative probability distributions. The analysis provides information that enables decision-makers to select the efficient RRA set that explicitly takes their attitude toward project risk into account. Decision-makers should find it both useful and practical for sound decision-making under uncertainty/risk and efficiently optimizing project success. The computations are readily performed using commercially available Monte Carlo simulation tools. The approach is detailed using a realistic but simplified case of a project with two technical risks.
\end{abstract}

Keywords: technical risk management, risk response actions, efficient set, probability of success, contingency, risk profile, risk trade-off, decision tree 


\section{INTRODUCTION}

In today's highly competitive environment and limited resources, the project manager ${ }^{1}$ needs to (1) cost the proposal low enough to win the project but high enough to ensure that the project is a success, and (2) once the project is underway, judiciously manage the technical and managerial risks. Project risks can be categorized as technical and managerial [Dillon and Paté-Cornell, 2001]. Technical risks are primarily associated with uncertainties in technology, performance, design, manufacturing, and integration. Managerial risks are associated with staff and management experience, inadequate schedule and budget, and programmatic issues. The unavailability of budget contingencies, when needed, results in schedule delays that further increase cost, scope changes, and/or project cancellation. It is no longer sufficient to just manage risk; the successful project manager must manage risk efficiently. Environmental risk management is another distinct element of risk management, and it is of critical importance for numerous industrial and R\&D projects [INCOSE, 1998: Section 4.5.3; Haimes, 1998]. The focus of this paper is on technical risks.

Risk analysis has a long and distinguished history [Bernstein, 1996]. Practitioners are beginning to recognize that risk analysis is not making as important a contribution to project management as it should because of the shortcomings of some of the more widely advocated approaches. Empirical studies [Shapira, 1995: 51] confirm that technical project managers want valid information rather than simplicity when making high-risk decisions. Chapman, Ward, and Bennell [2000] observe that decision-makers want "applied models that facilitate effective interventions" rather than inadequate theoretical models. Haymes [1998: 309] states: "One of the most dominant steps in the risk assessment process is the quantification of risk, yet the validity of the approach most commonly used to quantify risk - its expected value - has received neither the broad professional scrutiny that it deserves nor the hoped-for wider mathematical challenges that it mandates."

Numerous generic and statistical cost-risk models have been developed that use contingency factors [U.S. Department of Energy, 2000], cost estimating relationships [U.S. Department of Defense, 1999], and risk factors using the Analytical Hierarchy Process [Graham and Dechoretz, 1995]. These approaches provide a broad rather than detailed view of project risks, and consequently they do not provide the technical project manager with adequate guidance for planning and managing the risk reduction activities.

The present work focuses on the analysis and management of technical risks and presents an approach to balance risk, cost, performance, and schedule through the systematic development and implementation of Risk Response Actions (RRA). The selection of RRAs constitutes an important trade-off between how much to invest in the RRAs versus the level of risk to be assumed [Hillson, 1999]. The proposed approach provides a mathematically sound and practical solution to the following problem:

$>$ Determine the set of RRAs that either (1) maximizes the probability of success for a given total project cost, or (2) minimizes the total project cost for a given probability of success.

\footnotetext{
${ }^{1}$ The specific title of this role depends on the organization.
} 
The solution that we develop may be thought of as the analog of Markowitz' s "efficient frontier" [Markowitz, 1976] for project technical risks. We refer to it as the "efficient RRA set." It provides a variant of a couple of Markowitz' s "efficient portfolio selection" principles [Markowitz, 1976: 3] that we paraphrase as follows:

A good set of RRAs is more than a list of good individual RRAs. It is a balanced whole, providing the project with protections and opportunities with respect to a wide range of contingencies. If the RRA set is efficient, it is impossible to establish a smaller contingency without giving up some probability of success.

Markowitz's portfolio selection principles are powerful and they apply to areas beyond portfolio selection. For example, they have recently been extended to petroleum exploration and production [Ball and Savage, 1999].

Computing the efficient RRA requires evaluating discrete combinations of RRAs and determining the set that maximizes the probability of success for a given total project cost, or equivalently minimizes the total project cost for a given probability of success. The approach and its solution offer the following important benefits for risk management and resource allocation:

1. It links the RRAs to contingency planning and the probability of success. It can thereby be used to determine the lowest contingency cost required as a function of the assumed risk level.

2. It provides detailed information and visibility into the possible outcomes and selection of RRAs. It thereby supports the decision-makers' attitude toward risk and how they make real decisions.

3. It provides a mathematically sound and practical method that meets identified needs of decision-makers. Decision-makers should therefore find it useful.

We have selected to use various existing techniques including Decision Trees (DT), Monte Carlo simulation, and cumulative risk profiles to support and enhance the analysis process. To be specific:

1. We characterize risk in term of its cumulative distribution or risk profile. The proposed approach is therefore applicable to non-normal distributions that cannot be completely characterized by a mean and variance.

2. We do not rely on standard decision tree analysis that selects the option with the maximum or minimum expected outcome. The proposed approach thereby avoids the "fallacy of the expected value" [Haimes, 1999: 150] or the "flaw of averages" [Savage, 2000].

3. We quantify the possible outcomes in terms of monetary value and probability of occurrence. The proposed approach thereby avoids the difficulties associated with assessing and dealing with utility functions [Clemen and Reilly, 2001: Chapter 14].

4. We use scenarios to elicit the judgment and/or knowledge of experts about the possible outcomes and their level of confidence. The resulting data can be modeled using either discrete or continuous probability distributions.

5. The analysis can be readily implemented using commercial Excel ${ }^{\circ}$ add-ins $(@$ Risk $®$, Crystal Ball ${ }^{\circ}$, Insight.xla $\left.{ }^{\circledR}, \ldots\right)^{2}$ and/or more specialized tools (DecisionPro ${ }^{\circledR}$, Analytica $\left.{ }^{\circledR}, \ldots\right)^{2}$.

\footnotetext{
${ }^{2}$ This list is intended to be representative and not exhaustive.
} 
In this paper we detail the proposed approach for an individual project that contains multiple technical risks. The identified technical risks should be listed as individual risks only if they depend on distinct technical factors. It is therefore proper to model the technical risks as independent. For example, the SuperNova / Acceleration Probe (SNAP) mission [Kujawski, 2001] requires developing a space-based telescope with several scientific instruments (widefield imager, infrared photometer, spectrographs,...). These instruments face different technical challenges; the associated technical risks are independent; and the covariance between them is negligible. In Section 4.5, we briefly address the when and how to consider correlations between the individual risks.

The content of the paper is as follows. In the Introduction we presented the rationale behind the proposed approach and how we propose to build on and tailor some of Markowitz' s portfolio selection principles to apply to the management of project technical risks. In Section 2 we describe the use of scenarios to elicit risk information and risk profiles to characterize risk. In Section 3 we present the modeling and analysis of individual RRAs using the approach of Section 2. In Section 4 we develop a paradigm for determining the RRAs that make-up the efficient RRA set. The concepts of Sections 2, 3, and 4 form the basis upon which we select an efficient set of RRAs and determine an optimal total project cost contingency. In Section 5 we extend and apply these ideas to individual projects with multiple technical risks. In Section 6 we discuss the implications of the proposed approach to the management of technical risks and the planning and management of cost contingencies. In Section 7 we present some concluding comments. In the appendices we provide some additional details on modeling (Appendix A) and the illustrative examples (Appendix B).

\section{MATHEMATICAL MODELS FOR QUANTIFYING TECHNICAL RISK}

\subsection{Characterizing Risk}

We characterize risk by the probability and magnitude of the possible outcomes, referred to as its risk profile. We adopt the point of view that risk is too complex a concept to be fully captured by a single number [INCOSE, 1998: Section 4.5.3]. A full characterization of decision-making under uncertainty/risk requires specifying the following four elements: the possible events, their probability of occurrence, the range of impacts or outcomes associated with each event, and the conditional probability of each outcome given that the event has realized [Chapman and Ward, 1996]. This situation can be modeled in multiple ways, and in this work we use the following representations (See Appendix A for details):

1. Standard DTs using discrete outcomes.

2. Modified DTs where probability distributions rather than discrete branches are associated with the chance nodes. This provides the capability to model outcomes with continuous distributions and avoid bushy trees.

3. Spreadsheet-like models with explicit mathematical formulas. This provides a powerful technique to deal with complex situations and complements DTs.

\subsection{Using Scenarios to Quantify Technical Risk Contributors}


Scenarios [Chapman and Ward, 1996] provide a convenient technique to elicit the judgment of experts about probabilities and consequences of project technical risks. In the proposed method, we explicitly evaluate each risk contributor using three scenarios and a variation of the fractile method [Haimes, 1998] as follows:

1. Optimistic scenario or $20^{\text {th }}$ percentile of outcomes - It represents a credible upside scenario with a perceived probability of $20 \%$ (one chance in five) that the outcome could be better.

2. Pessimistic scenario or $80^{\text {th }}$ percentile of outcomes - It represents a credible downside scenario with a perceived probability of $20 \%$ that the outcome could be worse.

3. Most-likely scenario - It represents the scenario with the highest perceived likelihood of happening.

We use the "one chance in five" outcomes because these values are not too extreme and their use in place of upper and lower bounds assists the domain expert by raising useful questions of what could go wrong with the project baseline. Different levels of probability could be used [Markowitz, 1976: 32]. By specifying three outcomes and associated probabilities, the domain experts are able to encode their level of confidence for the possible outcomes. This data can be reported either in tabular or graphical form.

\subsection{Discrete versus Continuous Probability Distributions}

Cost and schedule exhibit a continuous range of outcomes. For this reason and possibly greater familiarity, analysts may opt to approximate the scenario data with continuous probability distributions. Depending on the statistical characteristics the analyst wants to capture, there is a multitude of ways to approximate a three-point discrete distribution by a continuous probability distribution. Popular parameterizations include the triangular and beta distributions [Garvey, 2000]. By explicitly starting with scenarios, the analyst can proceed to select the distribution that best characterizes the state of knowledge for the parameter or measure under consideration and avoid some of the shortcomings of the standard triangular distribution method [Moran, 1999].

\section{MODELING AND ANALYZING INDIVIDUAL RRAS}

There are typically many possible RRAs to a particular source of risk [Hall, 1998]. For the purpose of this paper, we explicitly consider the following three generic RRAs because they adequately categorize the available options with little or no loss of generality:

1. Accept the risk as is - The project recognizes the existence of the risk but considers it acceptable to simply monitor it using the standard approach.

2. Immediately implement risk reduction action - The project immediately implements RRAs such as selecting an alternate design, modifying the scope, switching vendors, pursuing parallel paths, etc. If desired, the project may simultaneously consider several of these approaches.

3. Obtain additional information - The project invests in additional analysis, testing, and/or prototyping before implementing costly RRAs. This approach provides a focus on dynamic risk management where effective RRAs are implemented as the situation evolves and information is acquired [Neely and de Neufville, 2001]. 
For the purpose of this paper we treat each RRA as a black box with only the inputs and outputs specified. As defined, the three categories of RRAs are mutually exclusive so that only one category of RRA is applicable at a given time for a given risk. But different RRAs can be simultaneously implemented for different risks within a project. The above RRA categorization can be further refined and readily implemented within the proposed approach. The only impact is to give rise to bigger models. In a given situation some of the RRAs may not be feasible because of technical and/or programmatic constraints. The model then includes fewer options and the DT reduces accordingly. Each project needs to develop and implement detailed risk mitigation / action plans for all medium and high risks. We use DTs as the framework for systematically developing and modeling the RRAs and associated decision process. But as discussed in Section 4, we do not use the standard DT analysis procedure of folding-back and averaging the outcomes for selecting the preferred RRAs.

\subsection{The Basic RRA Decision Tree}

We illustrate the approach by considering a single technical risk R1 with the above three generic RRAs as options. The resulting generic DT in Figure 1 lays out a simple architecture for developing and evaluating the RRAs for each individual risk. It also provides the basic template for dealing with multiple risks, as detailed in Section 5. Figure 1 follows the standard DT convention and the compact notation described in Appendix A. The three branches associated with the initial decision node represent the three RRAs identified above. The Obtain_R1_Data RRA sequence includes multiple decision nodes that reflect a staged decision situation where the subsequent decisions depend on the acquired information. The above basic DT is generic in character and readily generalizes to include more or fewer RRA options with the only impact being a bushier or leaner DT.

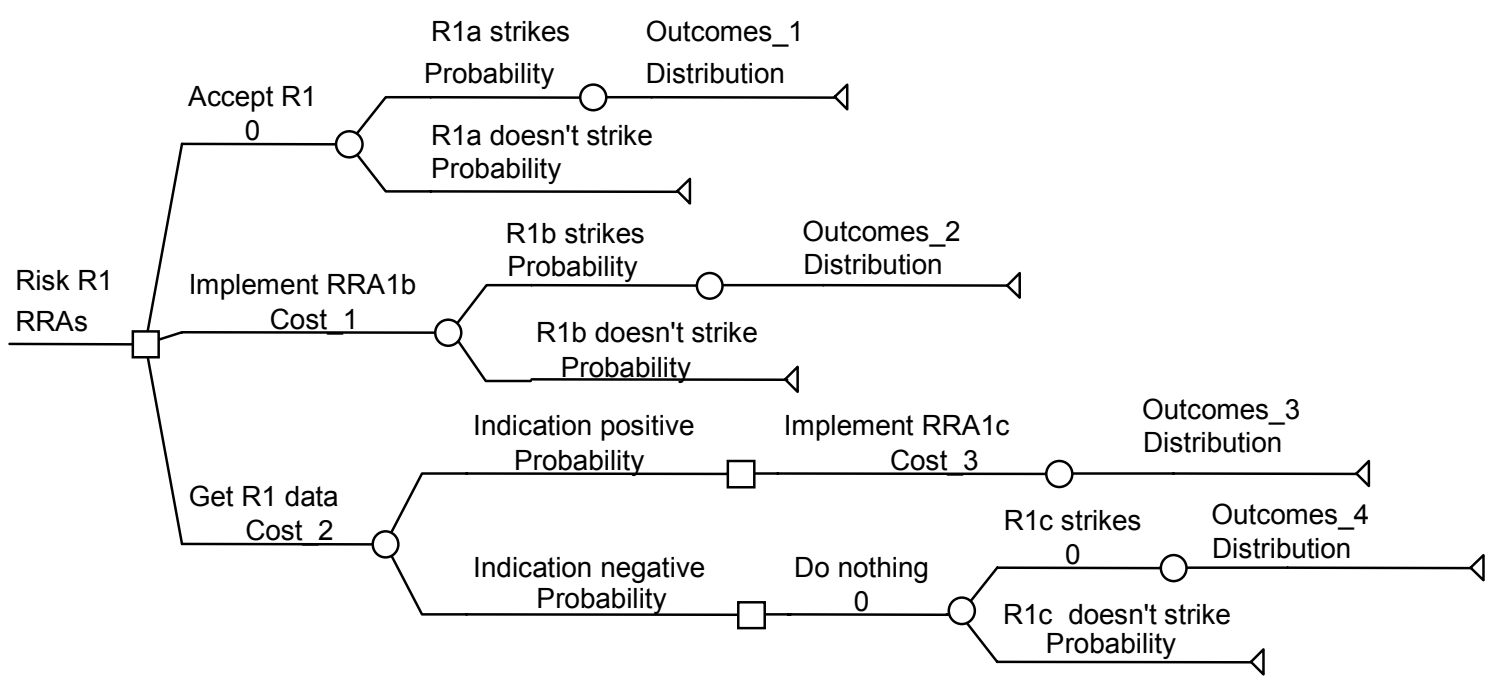

Figure 1. Basic RRA DT template.

\subsection{Quantification of the Basic RRA Decision Tree}

To proceed with the assessment of the RRAs, the variables of the RRA DT in Figure 1 need to be quantified. This is a challenging task that requires experience, the ability to make 
educated guesses, and a healthy dose of common sense. The probabilities and consequences associated with the Accept_R1 and Implement_RRA1 options should be directly obtained from the domain experts as described in Section 2. The values for the Get_R1_data RRA depend on the diagnostic capabilities of activities such as analysis, testing, and/or prototyping which, given finite resources, are not perfect. There is a tradeoff in deciding how much effort to expand on obtaining the additional information. A lower effort costs less, but it results in data that is less discriminating and a higher probability of a "false negative" that may result in severe consequences and higher costs later in the project. The data for the Get_R1_data RRA is determined as described in Appendix B.1.2.

\subsubsection{Illustrative Example: Project with a single technical risk}

We quantify the RRA DT in Figure 1 to reflect the following properties of realistic RRAs:

1. There is a cost associated with each RRA.

2. RRAs may incorporate a number of key decision points for evaluating progress and choose the subsequent best action. The Get_R1_data RRA represents such an option.

3. Practical RRAs are not $100 \%$ effective. Different outcomes are associated with each RRA. There is a cost associated with each residual risk.

4. The residual risks are less severe than the initial risks. The severe outcomes have both lower probabilities and consequences.

Figure 2 depicts the resulting mathematical model equivalent to the RRA DT in Figure 1.

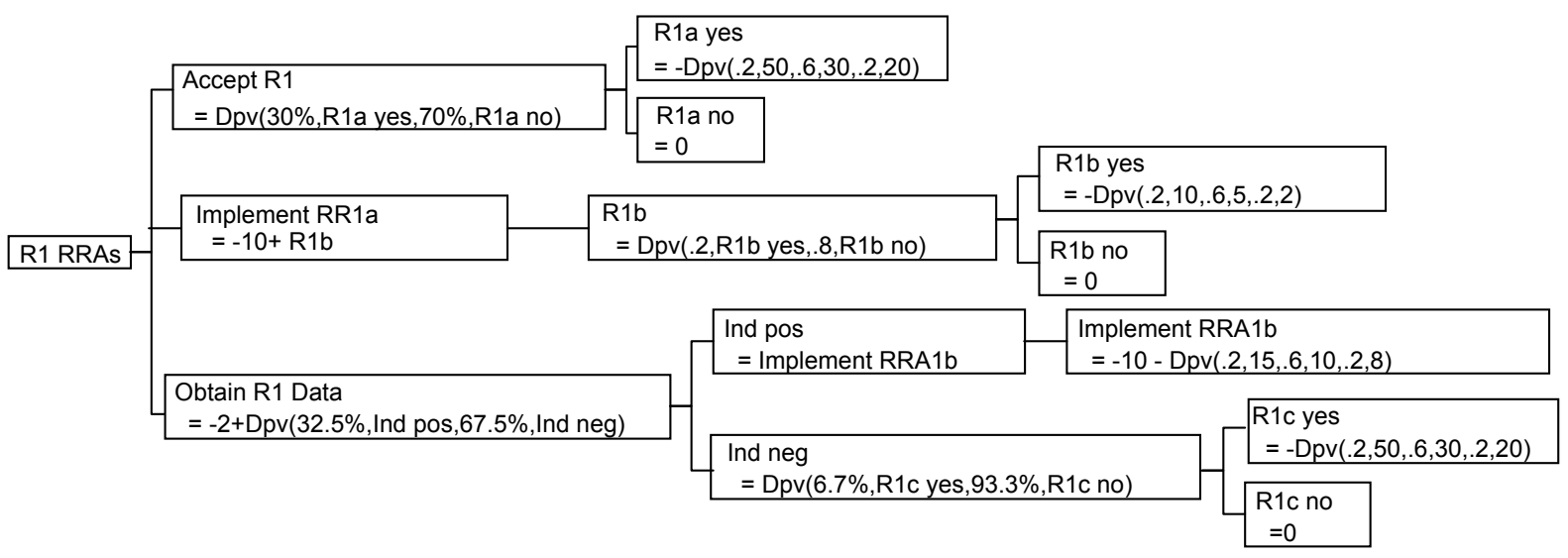

"R1a yes" is abbreviation for "R1a strikes"

"R1a no" is abbreviation for "R1a doesn't strike"

Dpv is the discrete probability distribution defined in Appendix B

Figure 2 Mathematical model for candidate R1 RRAs.

For ease of interpretation, each cell is identified with a self-descriptive label $^{3}$ and its mathematical formula. For completeness and assistance to the interested reader, we also provide the detailed quantified RRA DT in Appendix B. The choice of a representation is a matter of personal choice. We use the mathematical representation to emphasize that the proposed decision process does not rely on standard DT analysis (See Section 4.4).

\footnotetext{
${ }^{3}$ Some labels have been abbreviated for legibility.
} 
We evaluate the mathematical model in Figure 2 using Monte Carlo simulation because of its general applicability and convenience. The risk profiles and cumulative risk profiles for the individual RRAs are shown in Figures 3a and 3b, respectively.

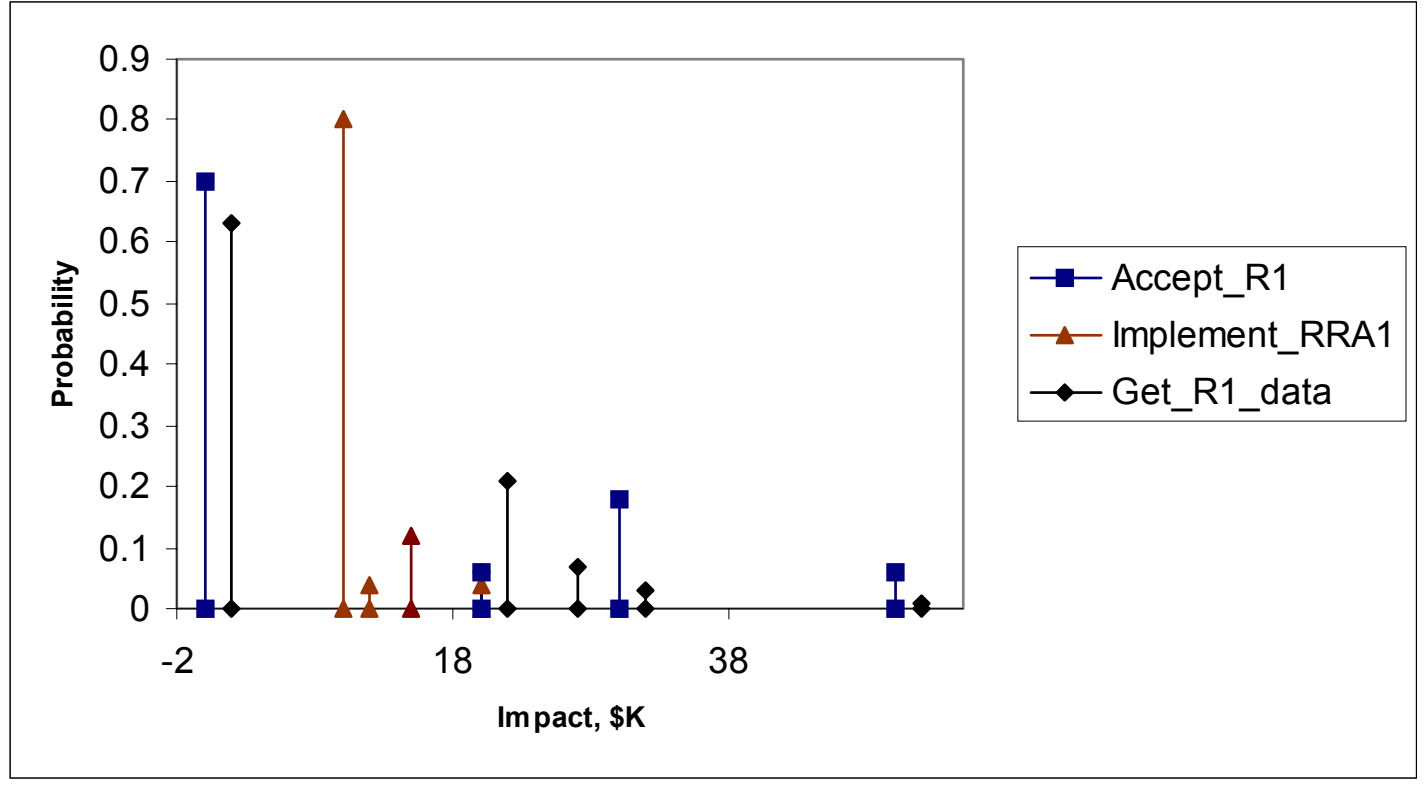

Figure 3a. Risk profiles for candidate R1 RRAs.

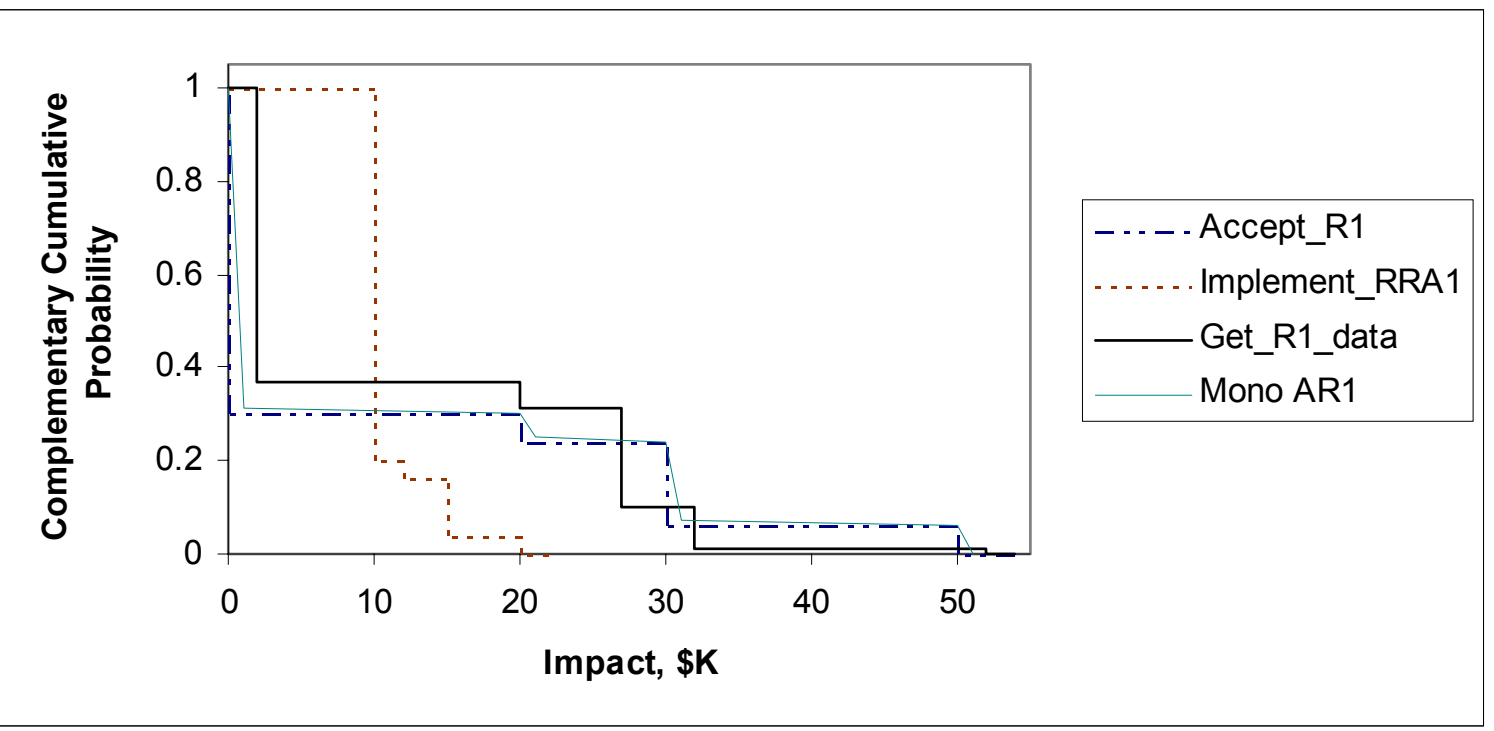

Figure 3b. Cumulative risk profiles for candidate R1 RRAs. 
The complementary cumulative probability is the probability of exceeding a given "impact" or 1-minus the probability of success ${ }^{4}$. Mathematically speaking, Figure $3 \mathrm{~b}$ plots 1 -minus the cumulative distribution function [Martz and Waller, 1982]. Given that we are interested in real-world projects, it is reasonable to approximate the step-like cumulative risk profile by a smooth function. For example, the curve "mono AR1" in Figure $3 b$ is such an approximation to the curve "Accept_R1". The associated means and standard deviations are compared in Figure 4. The reported results were obtained using Decision Pro ${ }^{\circledR}$. The Monte Carlo simulation converges rapidly within a couple of thousand trials that take only a few seconds on a Pentium ${ }^{\circledR}$ II PC.

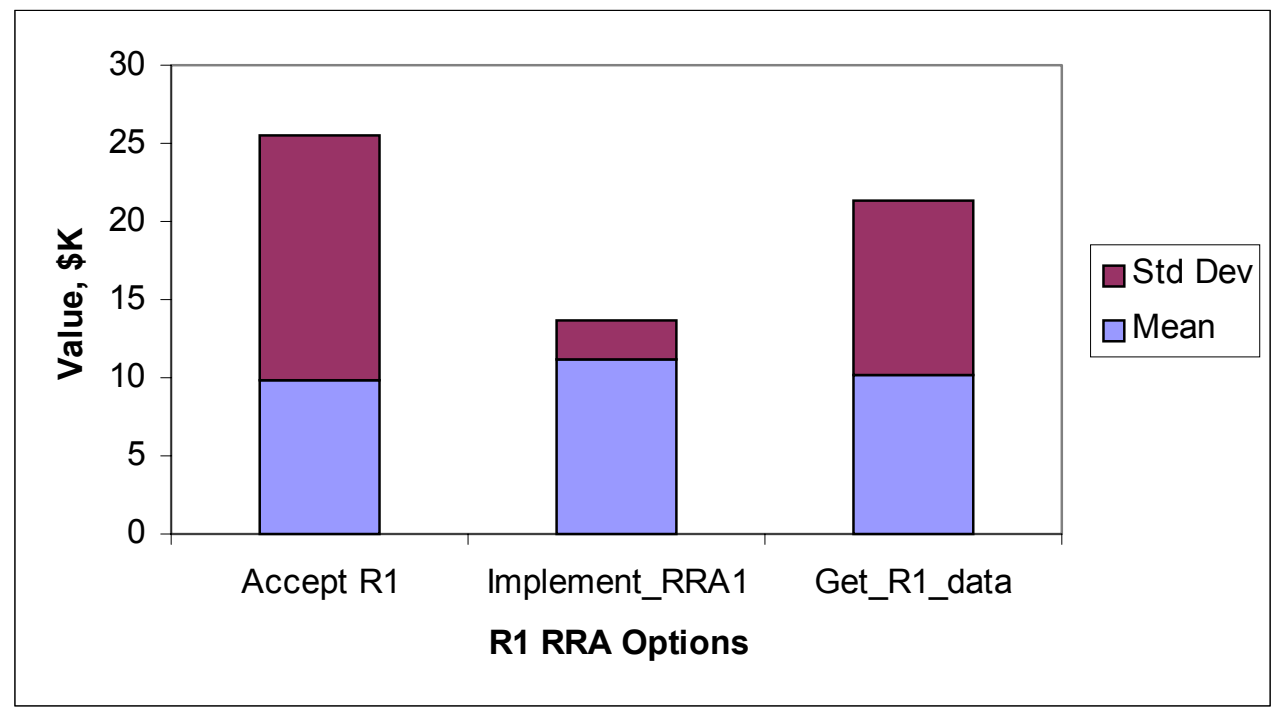

Figure 4. Means and standard deviations for candidate R1 RRAs.

Essentially identical results were obtained using other commercial tools (See Section 1). When using the RRA DT model, we individually simulate the subtree associated with each RRA. The selection of a RRA is a deterministic event and only the associated outcomes can be realized. It would therefore be inappropriate to weigh or combine the outcomes of the three RRA options since they are mutually exclusive events ${ }^{5}$. The simulation of DTs is further discussed in Appendix B.1. Finally, we point out that for this specific example that is based on discrete probability distributions, we could also have used the calculus of discrete probability distributions [Kaplan, 1981].

\section{DETERMINATION OF THE EFFICIENT RRA SET}

The risk profiles in Figure $3 \mathrm{a}$ and/or the cumulative risk profiles in Figure $3 \mathrm{~b}$ provide complete information and visibility into the range and probability of the possible outcomes for each RRA. But mathematically assessing such data is quite complex, and it is an active research area in many different fields including economics, safety, and psychology [Kahneman, Slovic, and Tversky, 1999]. We now examine the applicability of several techniques for comparing risks and determining the efficient RRA set. For illustration

\footnotetext{
${ }^{4}$ This observation was provided by one of the referees.

${ }^{5}$ Rob Suggs (Vanguard Software) provided this insight into the simulation of decision trees.
} 
purposes we use the data in Section 3. There is no loss of generality because the cumulative risk profile characterizes risk whether made up of a single risk or a project-wide set of multiple risks.

\subsection{Comparing Cumulative Risk Profiles}

Each cumulative risk profile in Figure $3 \mathrm{~b}$ provides the probability that the associated RRA does not exceed a given cost. For example, the Get R1 data RRA has a 63\% probability of costing $\$ 2 \mathrm{~K}$ or less and only a $0.9 \%$ probability of costing as much as $\$ 52 \mathrm{~K}$ on the high side. Such data is very valuable because it provides the project manager with the information needed to determine how much contingency should be available for a given confidence level or probability of success. The importance of such information is supported by empirical studies [Shapira, 1998] that show that the majority of technical project managers think that risk is not adequately characterized by its mean value but that it depends more on the magnitude than the probability of the undesirable outcomes.

The three cumulative risk profiles in Figure $3 \mathrm{~b}$ intersect each other. Intuitively, this means that no RRA (Accept_R1, Implement_RRA1, or Get_R1_data) is preferred under all possible selection criteria. In the language of the field, none of these RRAs exhibits "first order stochastic dominance" [Biswas, 1997]. The Implement_RRA1 RRA provides the lowest cost approach for achieving a probability of success exceeding $80 \%$. This cost ranges from $\$ 10 \mathrm{~K}$ at $80 \%$ to $\$ 22 \mathrm{~K}$ for essentially $100 \%$ probability of success. The two other RRA options cost more to provide the same probability of success. The Accept_R1 option provides a $70 \%$ probability of success for free; but it carries significantly higher risks than the other two RRAs. The Get_R1_data RRA provides a more deliberate approach between risk and a competitive project proposal cost. The preferred RRA depends on the decisionmaker's attitude toward risk; but as we demonstrate below there is a preferred solution for each decision-maker.

\subsection{Developing the Efficient Contingency Frontier}

When selecting RRAs from the set of available RRAs there is a subset of RRAs that provides a given probability of success for a lowest cost. This set of RRAs determines the lowest contingency necessary to support the acceptable probability of success. Its composition changes with the probability of success. We refer to the resulting set of RRAs as the "Efficient RRA Set" (ERRAS) and the associated points as the "Efficient Contingency Frontier" (ECF).

In this section, we illustrate the development of the ECF using the illustrative example in Section 3. We plot the outcomes associated with each RRA as points on the "Impact vs. Complementary Cumulative Probability" plane. The ECF is the curve that joins the points corresponding to the RRAs that provide the lowest cost impact for a given probability of success. Figure 5 depicts the result. 


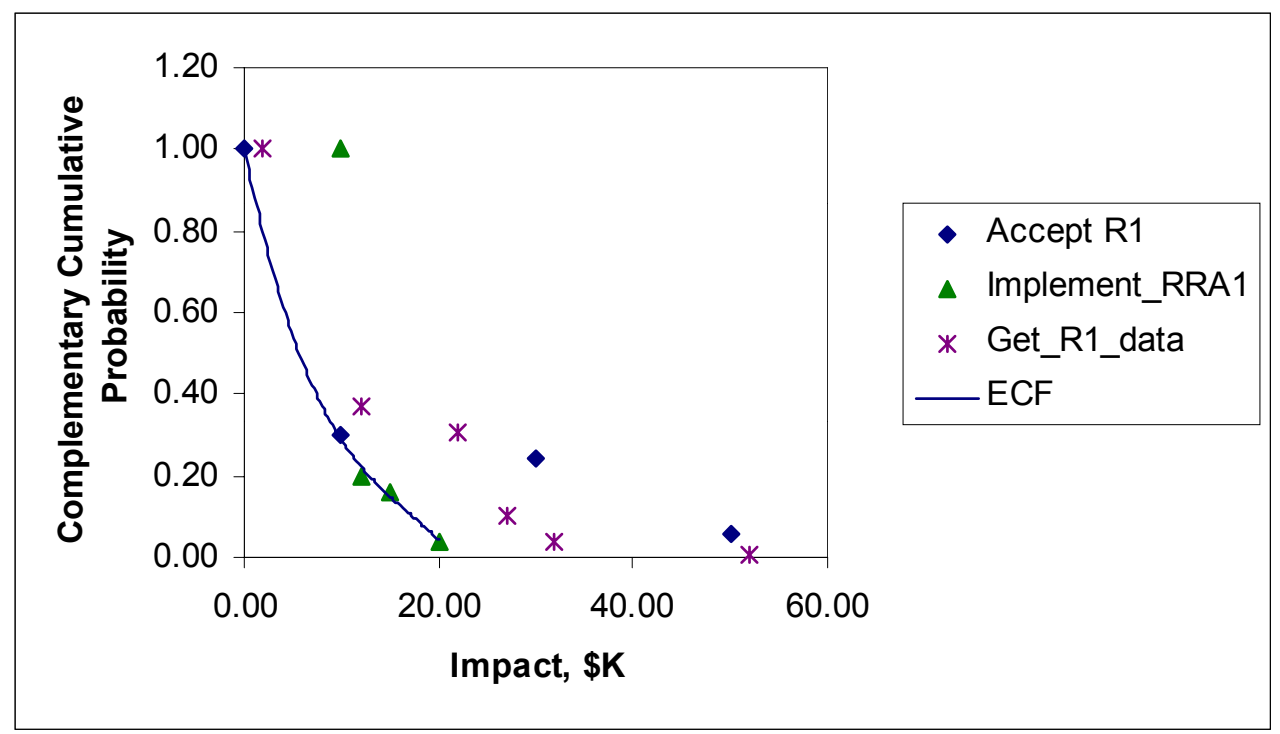

Figure 5. Determination of the efficient contingency frontier.

The points that lie to the right of the ECF represent RRAs that bear a greater cost impact than necessary for a given probability of success. None of the considered RRAs has a (Impact, Probability) represented by a point below the ECF. More formally, the ECF can be defined as the outer envelope of all the cumulative risk profiles (See Figure 14). To optimize winning the project and successfully carrying it out, project managers should specify the risk they are willing to take and then use the ECF to determine the contingency they need. Only the options that lie along the ECF need to be given further consideration. They represent Pareto-optimal solutions [Sen and Yang, 1998]. Points off the ECF represent inferior or dominated solutions.

\subsection{Applicability of Simple Metrics and Other Criteria}

Financial stocks can be adequately characterized using a few key statistical parameters such as the mean and variance [Markowitz, 1976]. But there are significant differences between financial portfolios and technical project risks that may invalidate the use of these two parameters for the latter. Project cost and schedule risk profiles are typically highly skewed [Schragenheim and Dettmer, 2001]; applicable statistical data are rarely available; and the focus is on the downside of individual projects. We use the example in Section 3 to illustrate the limitations of several simple metrics for applicability to technical risk analysis and contingency management. We also briefly examine how the analysis simplifies when the analyst can invoke the Central Limit Theorem [Garvey, 2000].

\subsubsection{Mean and Standard Deviation}

Figure 4 depicts the means $(\mu)$ and standard deviations $(\sigma)$ of the R1 RRAs. The Accept_R1 RRA has the smallest mean risk, $\$ 9.0 \mathrm{~K}$, and the largest standard deviation, $\$ 14.6 \mathrm{~K}$. In contrast, the Implement_RRA1 RRA has the largest mean risk, $\$ 11.0 \mathrm{~K}$, and the smallest standard deviation, $\$ 2.2 \overline{\mathrm{K}}$. Most technical project managers would agree that the Accept_R1 RRA represents a higher risk than the Implement_RRA1 RRA. This illustrates the fact that 
the mean or the standard deviation, as stand-alones, are not appropriate measures of risk for non-normal distributions.

\subsubsection{One-Standard-Deviation Value, $\mu-\sigma$}

\subsubsection{General Risk Distribution}

For normal distributions the value $(\mu-\sigma)$ has a cumulative probability $\operatorname{Pr}($ Value $<=$ $\mu-\sigma)=16 \%$. But for skewed distributions this probability needs to be explicitly calculated. For example, Figure $3 \mathrm{~b}$ provides the following values for the R1 RRAs:

- The Accept_R1 RRA has a 27\% probability of a cost greater than $\$ 24 \mathrm{~K}, \operatorname{Pr}(\operatorname{Impact}>24)$ $=27 \%$.

- The Implement_RRA1 RRA has a 37\% probability of a cost greater than $\$ 13 \mathrm{~K}, \operatorname{Pr}(\operatorname{Impact}$ $>13)=37 \%$.

- The Get_R1_data RRA has a 31\% probability of a cost greater than $\$ 21 \mathrm{~K}, \operatorname{Pr}(\operatorname{Impact}>$ 21) $=31 \%$.

The above data do not provide a clear ranking for the three RRAs. This illustrates the need for caution when using the one-standard-deviation value as the decision/selection criterion since its use can be dangerous when dealing with skewed distributions.

\subsubsection{Applicability of the Central Limit Theorem (CLT)}

The CLT [Garvey, 2000: 186] states that "The sum of a large number of independent random variables, where each makes a small contribution to the sum and its variance, approaches the normal distribution." When these conditions are satisfied, the total project contingency for any probability of success $\alpha$ is simply given by

$$
\mathrm{V}_{\alpha}=\Sigma \mu_{\mathrm{i}}-z_{\alpha} *\left(\Sigma \sigma_{\mathrm{i}}^{2}\right)^{1 / 2}
$$

where the sums range over the risks with means $\mu_{\mathrm{i}}$ and standard deviations $\sigma_{\mathrm{i}}$, and $z_{\alpha}$ is the standardized $z$-value from the normal distribution table [Shaikh, 1998]. The efficient RRA set is then determined by the values of $\mu_{\mathrm{i}}$ and $\sigma_{\mathrm{i}}$. This suggests that the analysis may simplify and be done analytically as the risks increase in number. However, the validity of invoking the CLT depends on the application and caution is required [Buck, 1989: Chapter 5].

We also see that when the CLT is applicable, the proposed approach reduces to a trade of "Expected cost vs. Variance of cost". This provides a formal link between the proposed approach and Markowitz's "Expected return vs. Variance of return" selection principle [Markowitz, 1976].

\subsubsection{Other Risk Criteria}

Technical project managers who are highly risk-averse tend to focus on worst-case scenarios and select the option with the lowest risk at a high confidence level. The $95^{\text {th }}$ percentile values ( $5 \%$ probability of a worse outcome) for the three RRAs in Figure $3 \mathrm{~b}$ are as follows: $\sim \$ 30 \mathrm{~K}$ for the Accept_R1 RRA; $\sim \$ 15 \mathrm{~K}$ for the Implement_RRA1 RRA; and $\sim \$ 22 \mathrm{~K}$ for the Get_R1_data RRA. Based on this criterion, the Implement_RRA1 RRA is preferred. But comparing risks using a single point on the cumulative risk profiles is not a robust method and does not provide a balanced consideration of all the possible outcomes. 
Risk aversion may be theoretically modeled using a utility function [Keeney and Raiffa, 1976]. These functions associate utilities or utiles with monetary values that reflect the decision-maker's certainty equivalent based on a "reference gamble or lottery." Accordingly, it is appropriate [Raiffa, 1970: 86] " to associate with each lottery its expected value in these new units and to choose the lottery that has the best showing on this new scale of expected values." But, empirical studies indicate that most technical managers are unlikely to use such data for decision-making under risk/uncertainty. To quote [Shapira, 1995: 51]: "Ideally, it would be an advantage for managers if risk could be described in one number. However, acknowledging the many facets of risk, most felt that transforming a multidimensional phenomenon to one number might not be adequate or helpful."

The Partitioned Multiobjective Risk Method (PMRM) [Haimes, 1998 and 2000; Gonick, 1996] is an interesting approach proposed for quantifying high consequence accidents. It characterizes risk using multiple conditional expected-value functions each associated with a particular range of damage severity. It thereby avoids decision-making based on the expected value.

\subsection{Limitations of Standard Decision Tree Analysis}

For completeness and clarity, we outline what in this paper we refer to as standard DT analysis [Clemen and Reilly, 2001]. It consists of the following steps:

1. Structure the decision and model it using a DT.

2. Quantify the outcomes or consequences at the end of the branches. The values may be physical quantities such as monetary values or subjective values based on a utility function.

3. Apply the "folding back the tree" procedure.

4. Choose the alternative with the highest or lowest expected outcome value.

Much of the valuable information obtained in the first two steps is subsequently lost in the subsequent steps. Since as indicated in the previous section technical managers do not favor utility theory or the use of expected value for decision-making under risk/uncertainty conditions [Shapira, 1995], we do not use standard DT analysis for determining efficient RRAs.

\subsection{Covariance and Correlation}

The importance of the covariance and correlation among the risk elements depends on the application, and it needs to be examined on a case by case basis. As indicated in Section 1, major technical risks within a single project typically depend on different risk factors and consequently they can be modeled as independent. Covariances are essential for security portfolios because the security returns and risks are strongly influenced by a common factor, the state of the economy. Covariance among the securities can then be related to various market indices [Markowitz, 1976: 98; Sharpe, 2000: 177]. For reliability and safety issues, it is very important to consider common-cause failures [Kujawski, Jacobs, and Smith, 1987]. These introduce dependencies between redundant or back-up systems, and the failures of these systems can no longer be treated as independent. Common-cause failures often become 
major design drivers because they are likely to dominate the low probability, high consequence events. We think that staff and management experience, schedule and budget constraints, and programmatic issues may introduce elements analogous to the market indices for project risks. But as already indicated, we categorize these risks as managerial rather than technical and outside the scope of the present paper.

When random variables are correlated, it is important to include the correlations in the analysis. This can readily be done within the proposed approach. The overall concept is not changed, but it requires the Monte Carlo simulation to generate correlated random variables [Haas, 1999]. This feature is available in some of the commercial software including Excel ${ }^{\circledR}$ add-ins identified in Section $1^{6}$. The challenge is getting valid data or models for the correlation factors for input into the analysis. The importance of the covariances depends on the application, and it needs to be examined on a case by case basis.

\section{DEALING WITH MULTIPLE RISKS}

We now build on the previous sections to deal with projects that face multiple technical risks and require the implementation of multiple RRAs. The objective is to determine the combination of RRAs that either (1) maximizes the probability of success for a given total project cost, or (2) minimizes the total project cost for a given probability of success. We generalize the concept of the ERRAS to a portfolio of multiple risks and refer to it as the Efficient Total Project RRA Set (ETPRRAS). The ETPRRAS includes multiple RRAs, and the Total Project ECF (TPECF) specifies the lowest Total Project Cost Contingency (TPCC) for a given probability of success. Formally, the problem can be stated as follows:

Given a project with multiple technical risks, $R_{i}$ where $(i=1, \ldots N)$, and associated RRAs, $R_{R A} A_{i j}$ where $\left(j=1,2, \ldots, N\right.$ for a given $\left.R_{i}\right)$, determine the combination of RRAs, $\left\{R R A_{1 j}\right.$, $\left.\mathrm{RRA}_{2 \mathrm{k}}, \ldots, \mathrm{RRA}_{\mathrm{Nl}}\right\}$, that either (1) maximizes the probability of success for a given total project cost, or (2) minimizes the total project cost for a given probability of success.

In Section 5.1 we first illustrate the approach for a hypothetical project with two technical risks. We then generalize the procedure to determine the efficient RRA set for projects with any number of risks and RRAs.

\subsection{Illustrative Example: A Project with Two Technical Risks}

Consider a hypothetical project with two technical risks, R1 and R2. To be more concrete, the reader may identify R1 with the technical risks of developing the telescope mirror and R2 with the technical risks of developing the optical imager for an advanced space-based science mission. Either risk may strike independently and adversely impact the project. By analogy to reliability analysis, each risk represents a single point failure [McCormick, 1981]. We model the problem as follows:

1. Risks R1 and R2 represent independent events and their outcomes do not affect each other.

2. The three generic RRAs defined in Section 3 are feasible options for each risk. Different RRAs may be selected for the individual risks.

\footnotetext{
${ }^{6}$ For example: @Risk ${ }^{\circledR}$ and Crystal Ball $®$.
} 
3. The outcome values depend on the joint RRAs.

4. The R1 data and R2 data are provided in Section 3.2.1 and Appendix B.2, respectively.

Given the above assumptions, there are nine possible Total Project RRAs (TPRRA) given by the set of combinations $\left\{R_{R A} A_{1 i}, R_{R} A_{2 j}\right\}$ where $i$ and $j=1,2,3$ corresponding to the three possible individual RRAs (Accept_ $R_{i}$, Implement_RRA ${ }_{i}$, Get_ $R_{i}$ data). Figure 6 depicts the resulting mathematical model. For convenience, the TPRRAs are denoted by $\mathrm{JR}_{\mathrm{k}}$ where $\mathrm{k}=1, \ldots, 9$. The residual risk associated with each joint RRA is quantitatively given by a probability distribution that is the statistical sum of the contributing risk probability distributions. Since the risks are independent, we compute these sums using a Monte Carlo simulation that independently samples the individual distributions.

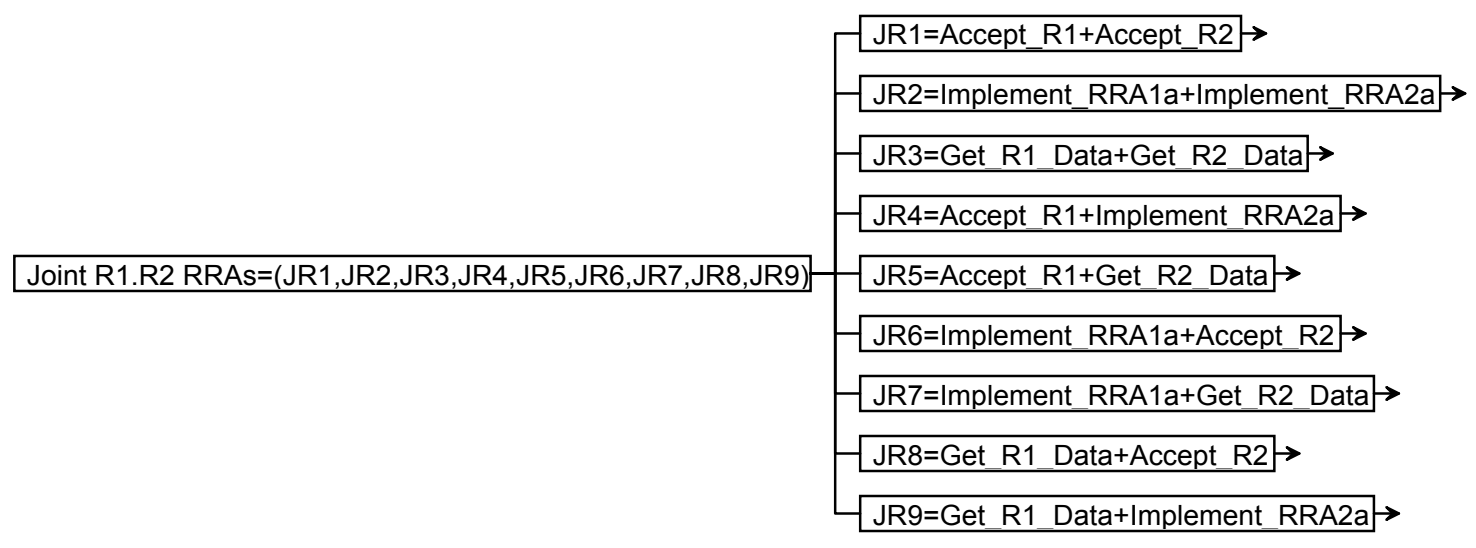

$\longrightarrow$ : Pointers to individual RRAs

Figure 6. Mathematical model for project with two risks.

The techniques of Sections 2, 3 and 4 are directly applicable to the total project risk, as summarized below:

1. Each total project RRA set is characterized in terms a cumulative risk profile and associated statistics.

2. The composition of the efficient total project RRA set varies with the probability of success and defines the total project ECF.

3. The total project ECF determines the lowest total project cost contingency for a given probability of success.

Figure 7 depicts the cumulative risk profiles of each of the nine joint RRAs. Given the space limitation and the resulting crowded appearance, it is intended for illustration purposes rather than detail. For convenience, we summarize a few key parameters including the mean, standard deviation, and selected outcomes in Table I. 


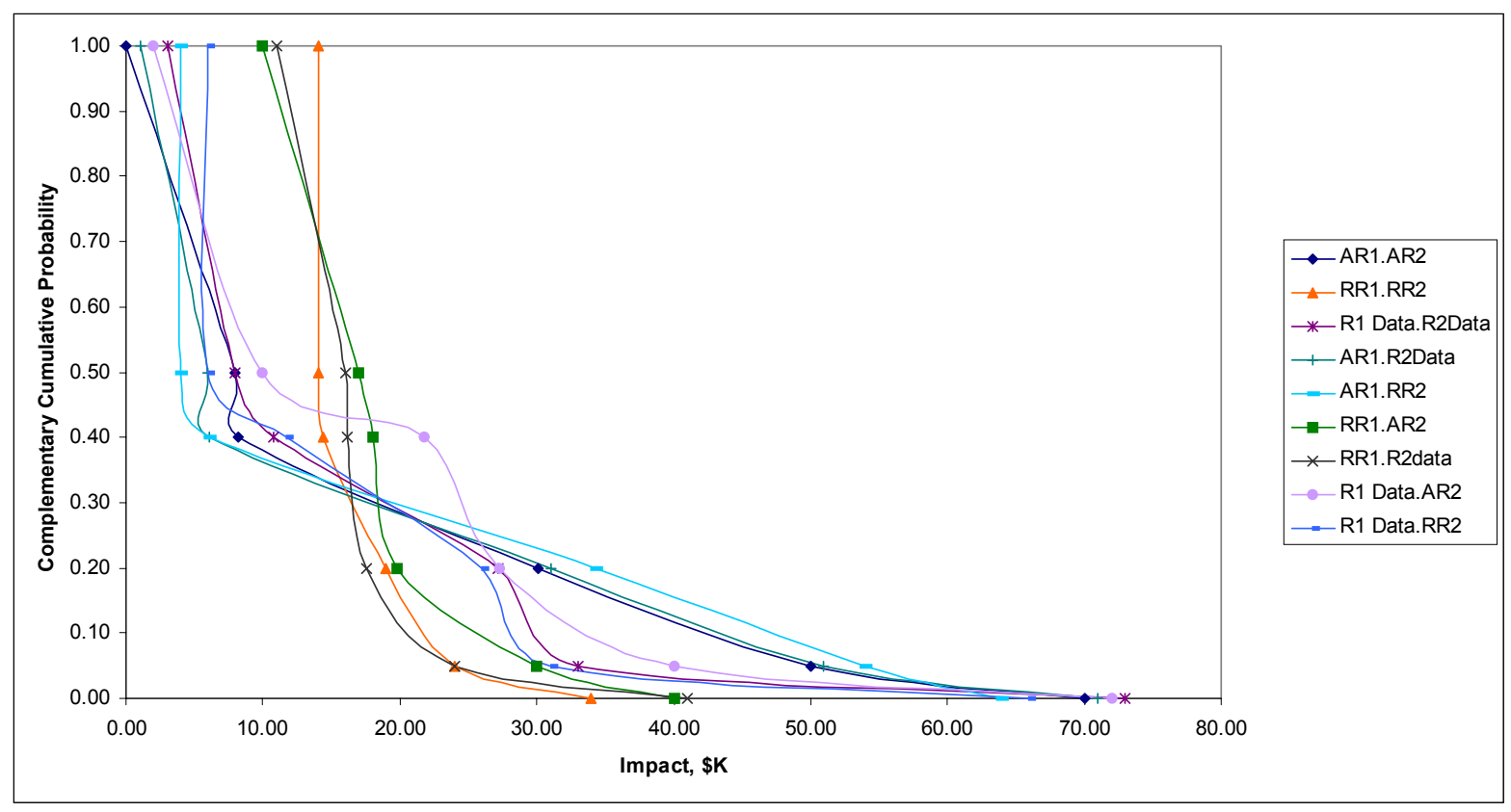

Figure 7. Cumulative risk profiles for project with two risks.

Table I. Summary Statistics for Project with Two Risks

\begin{tabular}{|c|c|c|c|c|c|c|c|}
\hline \multirow{3}{*}{ Project RRA* } & \multicolumn{7}{|c|}{ Cost Risk, \$K } \\
\hline & \multicolumn{7}{|c|}{ Probability of Success } \\
\hline & Mean & Std Dev & Min & Max & $50 \%$ & $80 \%$ & $95 \%$ \\
\hline JR1: AR1.AR2 & 15.1 & 16.9 & 0.0 & 70.0 & 8.0 & 30.1 & 50.0 \\
\hline JR2: RR1.RR2 & 15.9 & 3.3 & 14.0 & 34.0 & 14.0 & 19.0 & 24.0 \\
\hline JR3: R1Data.R2Data & 14.7 & 11.7 & 3.0 & 73.0 & 8.0 & 27.2 & 33.0 \\
\hline JR4: AR1.R2Data & 14.2 & 16.0 & 1.0 & 71.0 & 6.0 & 31.0 & 51.0 \\
\hline JR5: AR1.RR2 & 14.4 & 15.9 & 4.0 & 64.0 & 4.0 & 34.4 & 54.0 \\
\hline JR6: RR1.AR2 & 16.6 & 6.8 & 10.0 & 40.0 & 17.0 & 19.8 & 30.0 \\
\hline JR7: RR1.R2data & 15.7 & 4.3 & 11.0 & 41.0 & 16.0 & 17.6 & 24.0 \\
\hline JR8: R1Data.AR2 & 15.7 & 12.9 & 2.0 & 72.0 & 10.0 & 27.2 & 40.0 \\
\hline JR9: R1Data.RR2 & 15.0 & 11.3 & 6.0 & 66.0 & 6.0 & 26.0 & 31.0 \\
\hline
\end{tabular}

* See Figures 6 and 7

Based on these data, we make the following observations:

1. No total project RRA set dominates for all possible outcomes. The composition of the efficient total project RRA set varies with the probability of success.

2. The individual risk ECFs do not add algebraically. When assessing the impact of a joint RRA (RRAi for Risk \#1 and RRAj for Risk \#2) one must probabilistically (not algebraically) sum the impacts of RRAi and RRAj in order to determine the TPECF. This has important implications for project management and establishing the TPCC [Kujawski, 2002].

3. JR4, \{Accept_R1, Implement_RRA2a\}, has the lowest mean value $(\$ 14.2 \mathrm{~K})$ and one of the largest standard deviation $(\$ 16.0 \mathrm{~K})$. Most project managers would not select it as the preferred option. 
4. JR7, \{Implement_RRA1a, Get_R2_data $\}$, dominates for probabilities of success between $80 \%(\$ 17.6 \mathrm{~K})$ and $95 \%(\$ 24 \mathrm{~K})$.

5. JR2, \{Implement_RRA1a, Implement_RRA2a\} dominates for probabilities of success greater than $95 \%$ and has an estimated maximum cost of $\$ 34.0 \mathrm{~K}$.

6. The cost differentials among the different strategies are significant. For example, JR7, \{Implement_RRA1a, Get_R2_data\}, provides an 80\% probability of success for $\$ 17.6 \mathrm{~K}$ while the corresponding cost with JR5, \{Accept_R1, Implement_RRA2a\}, is $\$ 34.4 \mathrm{~K}$. The cost-benefit of judicious risk management increases with increased probability of success.

7. JR7, \{Implement_RRA1, Get_R2_data\}, provides an effective hybrid RRA option. Successful project managers often favor such options, where additional data is pursued for some of the risks.

As an aside, we note that for all of the above RRAs the value given by the sum of the mean and standard deviation corresponds to approximately an $80 \%$ probability of success. We find this somewhat surprising, but it may be another example where the CLT provides a good approximation even with a small number of risks. This is interesting because it suggests that the closed form risk value approximation presented in Section 4 may have wider applicability than one might expect [Buck, 1989: Chapter 5].

\subsection{Workflow for Determining the Efficient RRA Set and Optimal Contingency}

Figure 8 depicts the workflow that a project follows to develop its own project-specific RRAs in accordance with the approach developed in this paper.

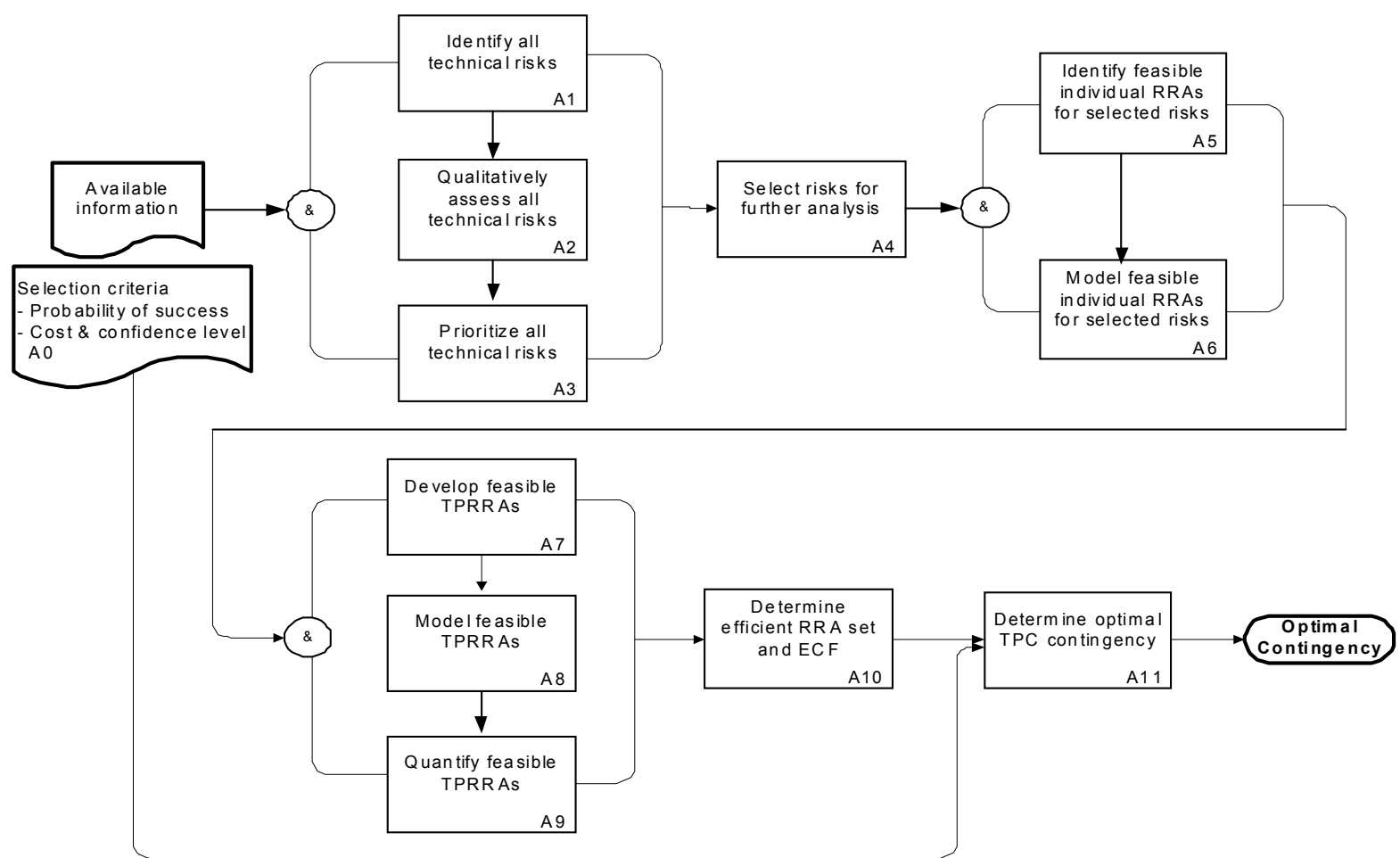

Figure 8. Workflow for determining the efficient RRA set and optimal contingency. 
The workflow for determining the efficient RRA set and optimal contingency consists of the following activities:

1. Specify the criteria for selecting the RRAs. These include the acceptable probability of success or Total Project Cost (TPC) and Confidence Level (CL). (Activity A0).

2. Identify, quantify, and prioritize all technical risks. Each risk should depend on distinct risk factors (Activities A1 - A3).

3. Select the significant risks for further analysis. (Activity A4).

4. Develop, model, and quantify the feasible individual RRAs. (Activities A5 - A6).

5. Develop, model, and quantify the feasible total project RRA Sets. (Activities A7 - A9).

6. Determine the efficient total project RRA set, the total project ECF, and the optimal total project contingency cost. (Activities A10 - A11).

As discussed in Section 3, some RRAs may not be feasible for a given risk. For illustration purposes we refer to the example in Section 5.1. If the Get_R2_data RRA were not feasible for risk R2 because of schedule constraints, then only six joint RRA options (those that do not include the Get_R2_data RRA) would need to be considered and evaluated to determine the total project ECF.

\section{IMPLICATIONS FOR RISK AND CONTINGENCY MANAGEMENT}

This paper addresses the following risk management questions:

1. How much contingency should be available to ensure the acceptable probability of success?

2. What set or combination of individual RRAs provides the acceptable probability of success for the lowest cost?

3. What are the risk profiles (probability vs. consequence) of the candidate total project RRA sets?

The proposed solution has significant implications for efficiently managing project risks and cost contingencies:

1. For every level of risk there is a combination of individual RRAs, the efficient total project RRA set, that provides the acceptable probability of success for the lowest cost. The composition of this set varies with probability and it defines the ECF or optimal total project contingency.

2. It is important to explicitly consider risk at the project-wide level. The total project efficient RRA set is not simply the combination of the individual efficient RRAs. There are other numerous examples in systems engineering where decomposition and reconstruction can cause serious problems [Friedman, 1999].

3. Selecting the total project efficient RRA set should be the primary objective of risk management. Higher risk should be viewed as a trade-off in trying to achieve a higher probability of project win vs. higher profit.

4. In many situations, the mean and variance do not fully characterize risk and their use may in some cases lead to incorrect decisions. Decision-makers need additional data that provide greater visibility into the individual and total project risk profiles.

5. The ability to determine the efficient total project RRA set empowers the decision-maker to manage risk and thereby achieve the highest probability of success for a given contingency. 
6. The total project contingency cost should be held centrally and managed at the projectwide level. This principle is essential to successfully manage the contingency and should be implemented as an integral element of the proposed approach [Kujawski, 2002].

\subsection{CONCLUDING COMMENTS}

The focus of the present work is on the technical risks associated with uncertainties in technology, performance, design, manufacturing, and integration. Effective risk management requires that the selection of RRAs be treated as a trade-off between how much to invest in risk mitigation versus the level of risk to be assumed, i.e. overall project cost vs. probability of success. We have developed a practical and mathematically sound approach for developing, selecting, and implementing the set of RRAs that achieves the lowest total project cost for a given probability of success. This set may be viewed as the project technical risk analog of Markowitz's efficient frontier for security portfolio, and we refer to it as the "efficient RRA set". It requires the smallest contingency for a given probability of success.

The proposed approach uses various existing techniques including decision trees, Monte Carlo simulation, and cumulative risk profiles. But it does not rely on standard decision tree analysis and the use of expected value for selecting the efficient RRA set. Scenarios are used to elicit and quantify risk data. Complex situations with both discrete and continuous outcomes are conveniently modeled using compact representations. Correlated risks can be modeled using Monte Carlo simulation or other approaches that generate correlated random variables. The analysis is readily implemented using commercially available software including some Excel ${ }^{\circledR}$ add-ins. The results provide detailed information and visibility that decision-makers need and want when they face high-risk decisions. In conclusion, we have developed a practical and mathematically sound approach that addresses the needs of technical managers who want valid information rather than simplicity for decision-making under uncertainty/risk.

The project manager who properly implements the proposed approach and principles to technical risk management is more likely to have a successful project at a lower cost. The challenges to the team are:

- Think of selecting the total project RRA set as a whole and not simply focus on the individual risks per se.

- Treat risk management as an integral part of project management.

- Adequately identify and quantify all the risks.

- Develop and quantify the individual RRA options.

\section{APPENDIX A: MODELING REPRESENTATIONS AND NOTATION}

Figure 9 depicts the modeling representations discussed in Section 2:

1. Figure 9a. A simple DT with each branch representing an individual outcome. There is no loss of generality by not considering decision nodes.

2. Figure 9b. A probability distribution is associated with each branch in the DT. In the case shown, the single branch with the specified discrete distribution function replaces 
the top four branches in Figure 9a. More generally, we use $\operatorname{Dpv}\left(\mathrm{P}_{1}, \mathrm{~V}_{1}, \mathrm{P}_{2}, \mathrm{~V}_{2}, \ldots, \mathrm{P}_{\mathrm{n}}, \mathrm{V}_{\mathrm{n}}\right)$ to denote the discrete distribution function with $n$ possible outcomes with value $V_{i}$ and probability $\mathrm{P}_{i}$ for outcome $i$. This representation readily generalizes to arbitrary distributions and provides a framework for modeling outcomes with continuous distributions.

3. Figure 9c. DTs can also be modeled using a standard spreadsheet as shown. The cells in Figure 9c contain formulas that are equivalent to the corresponding node and branch in the ET in Figures 9a and 9b. Such mathematical models provide a convenient framework for dealing with complex trees where the values of branches are defined by probability distribution functions.

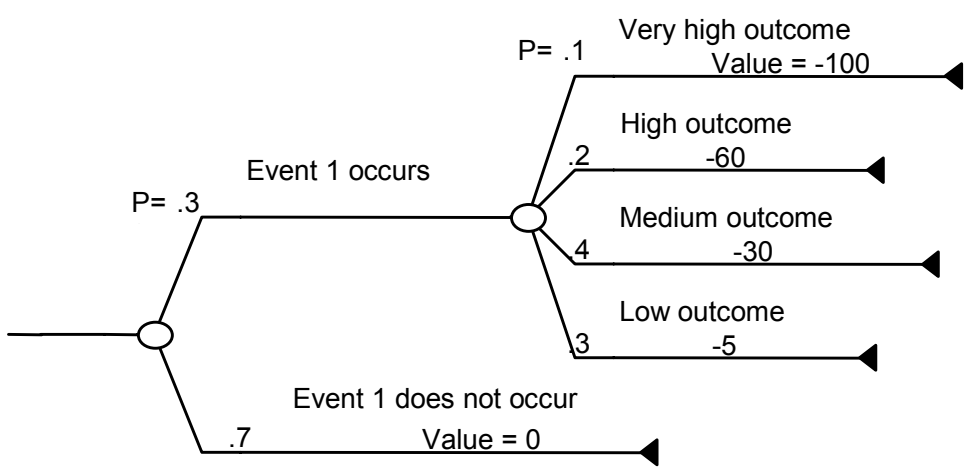

(a) Standard representation

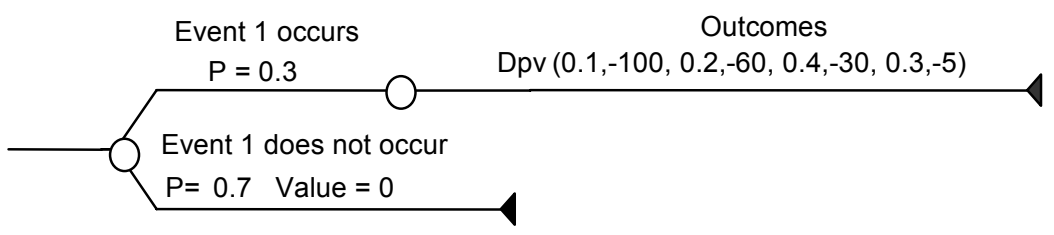

(b) Representation using discrete distribution function

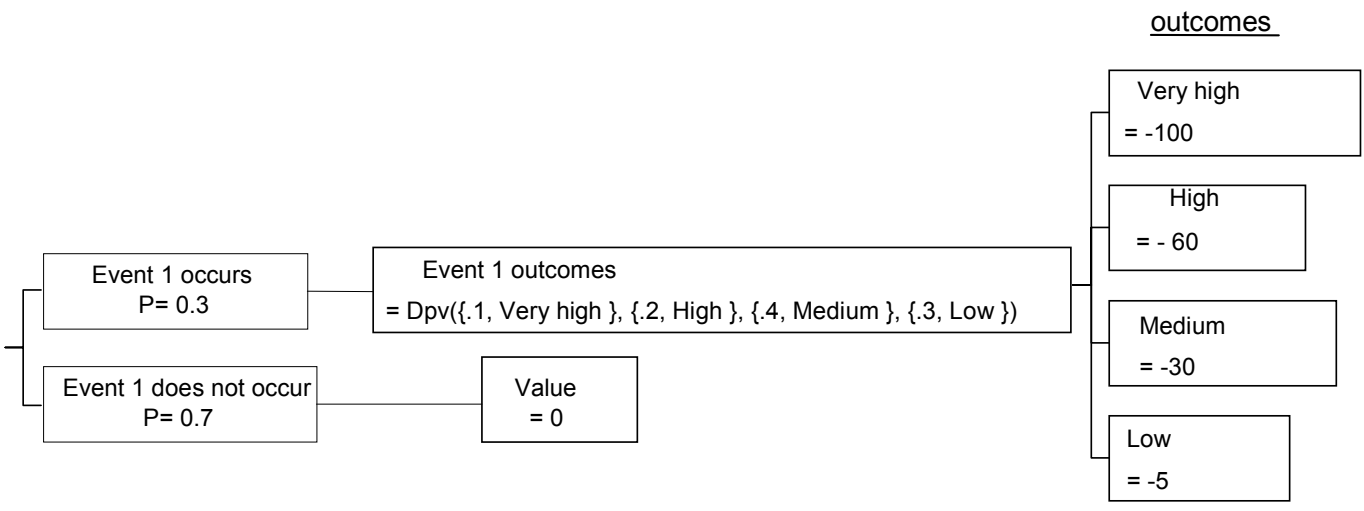

\section{(c) Spreadsheet model}

Figure 9. Example of a probabilistic scenario using different representations. 


\section{APPENDIX B: ADDITIONAL DATA FOR ILLUSTRATIVE EXAMPLES}

\section{B.1. R1 RRAS}

\section{B.1.1 Detailed RRA DT}

Figure 10 depicts the standard DT representation for the R1 RRAs described in Section 3.
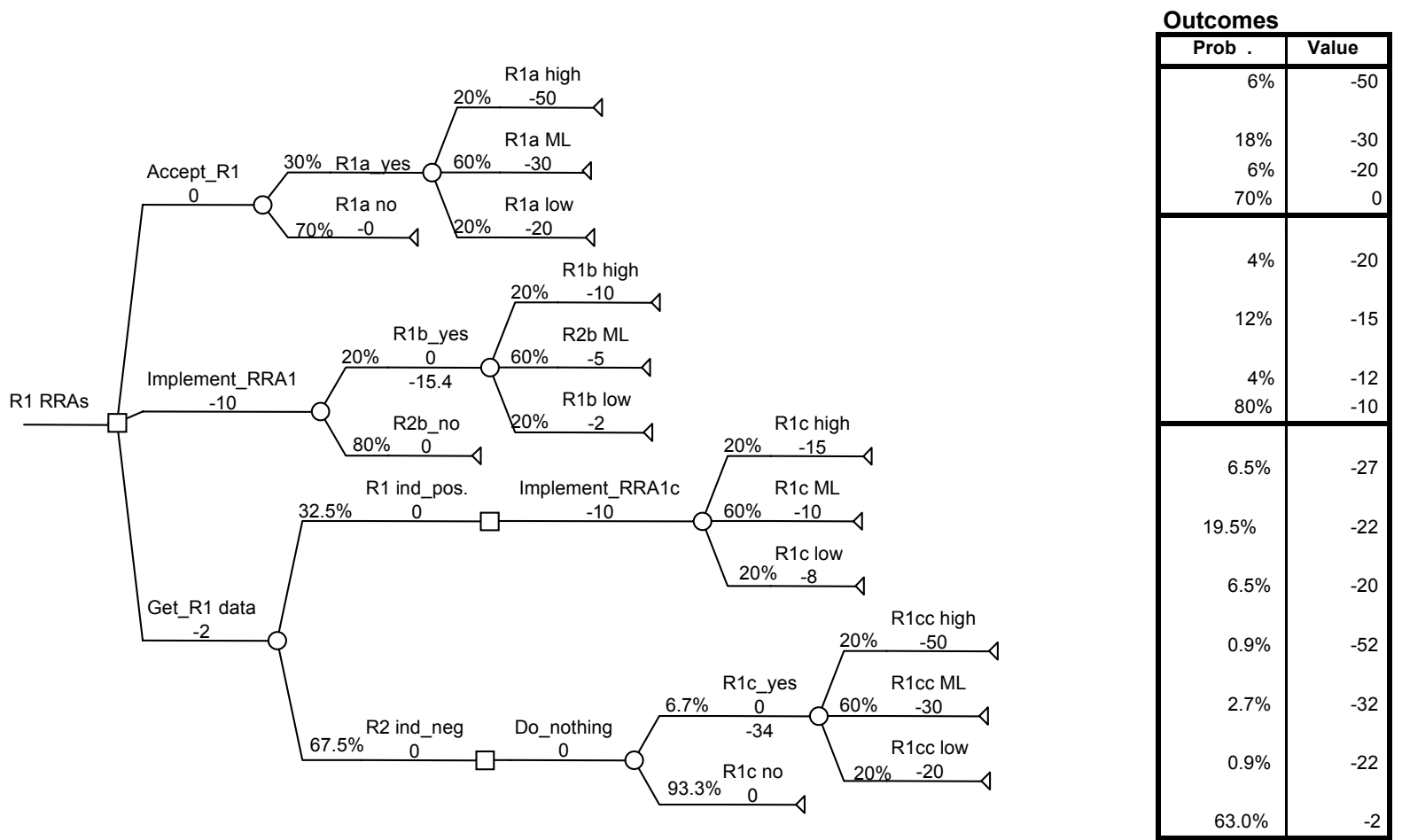

Figure 10. R1 RRAs using standard DT representation.

Decision nodes and chance nodes are depicted as squares and circles, respectively. The branches that originate with decision nodes represent the available RRAs. The branches that originate with chance nodes represent the possible probabilistic outcomes. Each branch has a probability and cost value associated it. These values are conditional on the RRA. To differentiate among the three RRAs, we explicitly denote the associated risks as R1a, R1b, and R1c.

The probabilities of the outcomes associated with each RRA sum to 1. This is an important check on the validity of any RRA DT model. The three RRAs are mutually exclusive, and each has its own and independent existence. Decision nodes are deterministic; only one of RRAs gets implemented for each risk. Once a RRA is implemented, only the associated outcomes can be realized and the sum of their probabilities must equal 1. To characterize each RRA, the subtree associated with each RRA needs to be analyzed individually. As it should be, the risk profile and cumulative risk profile for each RRA are identical to those shown in Figures $3 \mathrm{a}$ and $3 \mathrm{~b}$, respectively. 


\section{B.1.2. Quantification of the Get_R1_data RRA}

As indicated in Section 3, the Get_R1_data RRA requires additional analysis beyond elicitation of the data from the domain experts. The domain experts normally have information on the prior probabilities of $\mathrm{R} 1$ and the effectiveness of the proposed testing and/or analysis. The information obtained during the risk reduction phase may be erroneous and may result in any of four events. We assume that the following characteristics for both the Get_R1_data and Get_R2_data RRAs:

- Event 1: Given that the risk R1 (R2) is present, there is an $85 \%$ probability that the additional data detects it.

- Event 2: Given that the risk R1 (R2) is present, there is a $15 \%$ probability that the additional data does not detect it, i.e. gives a "false negative" indication.

- Event 3: Given that the risk R1 (R2) is not present, there is a 10\% probability that the additional data indicates that the risk is present, i.e. gives a "false positive" indication.

- Event 4: Given that the risk R1 (R2) is not present, there is a 90\% probability that the additional data indicates that it is not present.

The above four events for the R1 risk are depicted in Figure 11a. But these events appear in the reverse order of the events in the tree in Figure 10. In Figure 10 we first collect data and then use it to obtain a better estimate of the presence or absence of risk R1. Bayes' formula [Haimes, 1998] enables one to "reverse" the order of information gathering and compute the needed conditional probabilities. For completeness, we illustrate the calculation of the lowest branch:

$\operatorname{Pr}(\mathrm{R} 1$ present $\mid \mathrm{R} 1$ ind. neg $)=\operatorname{Pr}(\mathrm{R} 1$ present $) * \operatorname{Pr}(\mathrm{R} 1$ ind. neg. $\mid \mathrm{R} 1$ present $) / \operatorname{Pr}(\mathrm{R} 1$ where ind. neg),

$\operatorname{Pr}(\mathrm{R} 1$ ind. neg $)=\operatorname{Pr}(\mathrm{R} 1 \text { ind. neg. } \mid \mathrm{R} 1 \text { not present })^{*} \operatorname{Pr}(\mathrm{R} 1$ not present $)+\operatorname{Pr}(\mathrm{R} 1$ ind. neg. | R1 present)*P(R1 present).

Substituting the above specified values yields the following data for outcome \#3in Figure 11b:

$\mathrm{P}(\mathrm{R} 1$ ind. neg $)=0.9 * 0.7+0.15 * 0.3=67.5 \%$

$\mathrm{P}(\mathrm{R} 1$ present $\mid \mathrm{R} 1$ ind. neg $)=(0.3 * 0.15) / 0.675=6.7 \%$.

Figure $11 \mathrm{~b}$ depicts the "reversed event tree" that results from applying the above analysis to the event tree in Figure 11a.

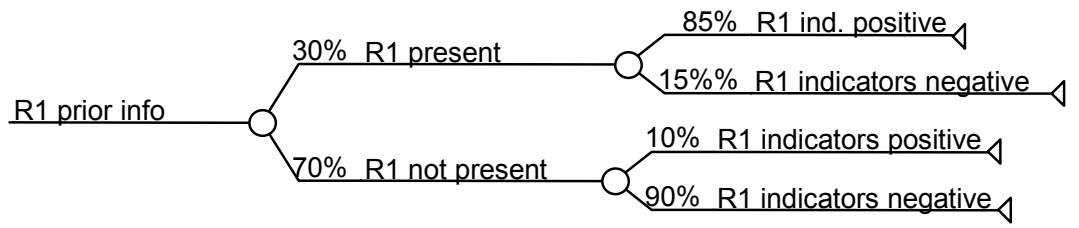

Figure 11a. Event tree based on prior information. 


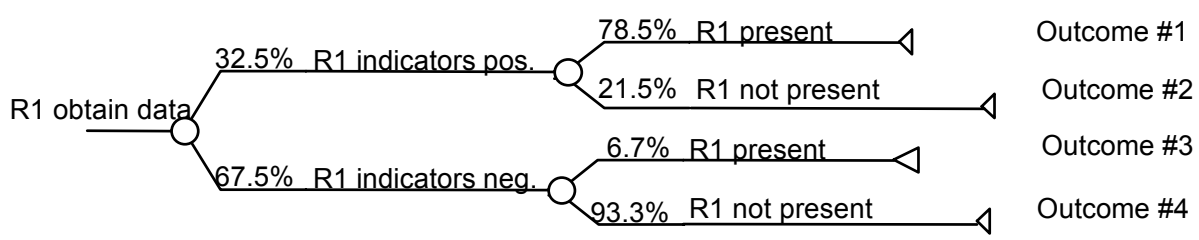

Figure 11b. "Reversed" event tree.

\section{B.2. R2 RRAs}

We quantify the R2 RRAs in a similar manner. The resulting data is summarized as follows:

- Figure 12 depicts the quantified mathematical model

- Figure 13 compares the means and variances of the three RRAs

- Figure 14 depicts the cumulative risk profiles and the associated ECF.

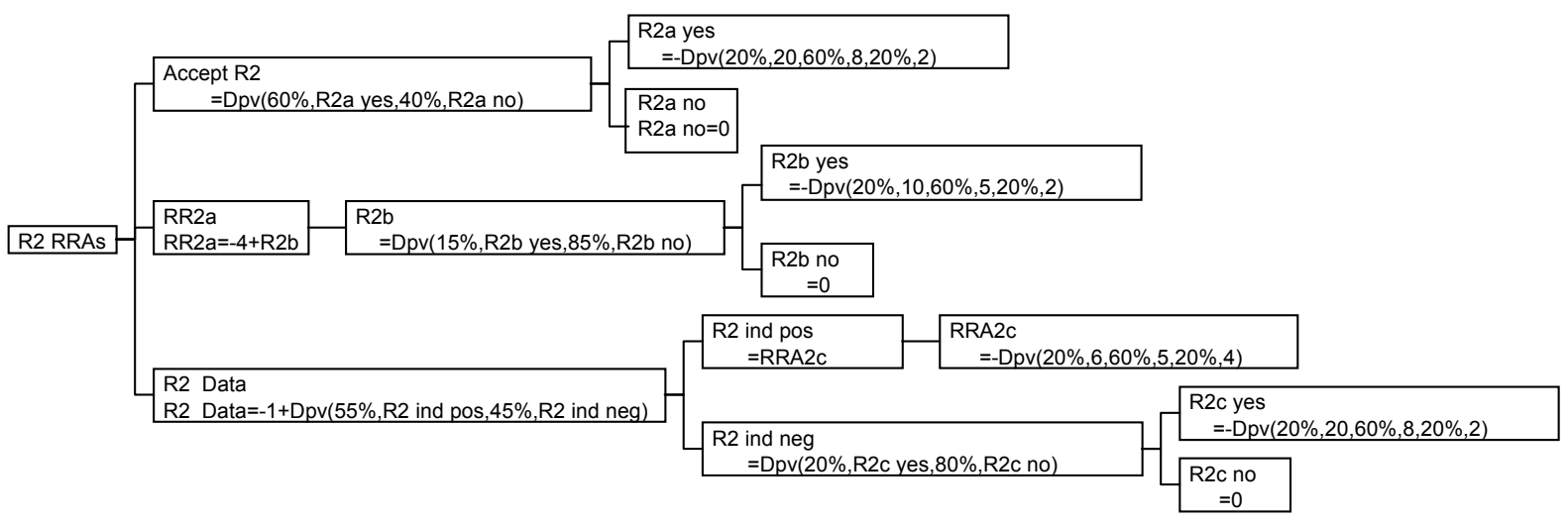

Figure 12. Quantified mathematical model for R2 RRAs.

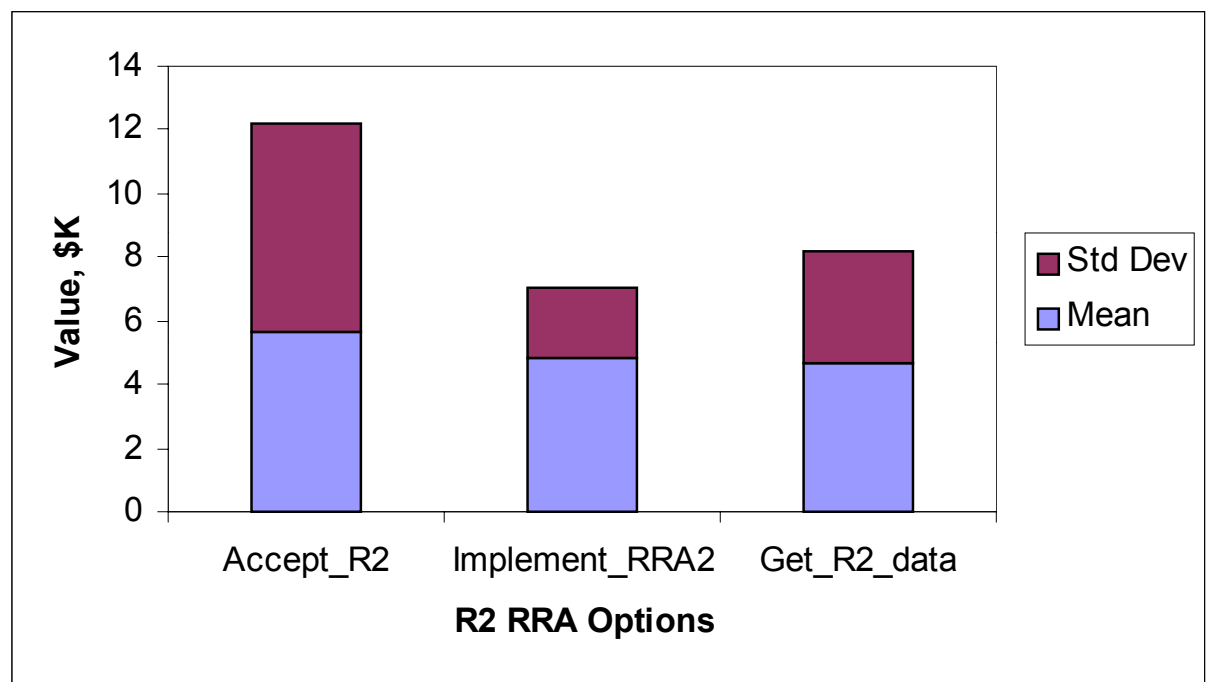

Figure 13. Means and standard deviations for R2 RRAs. 


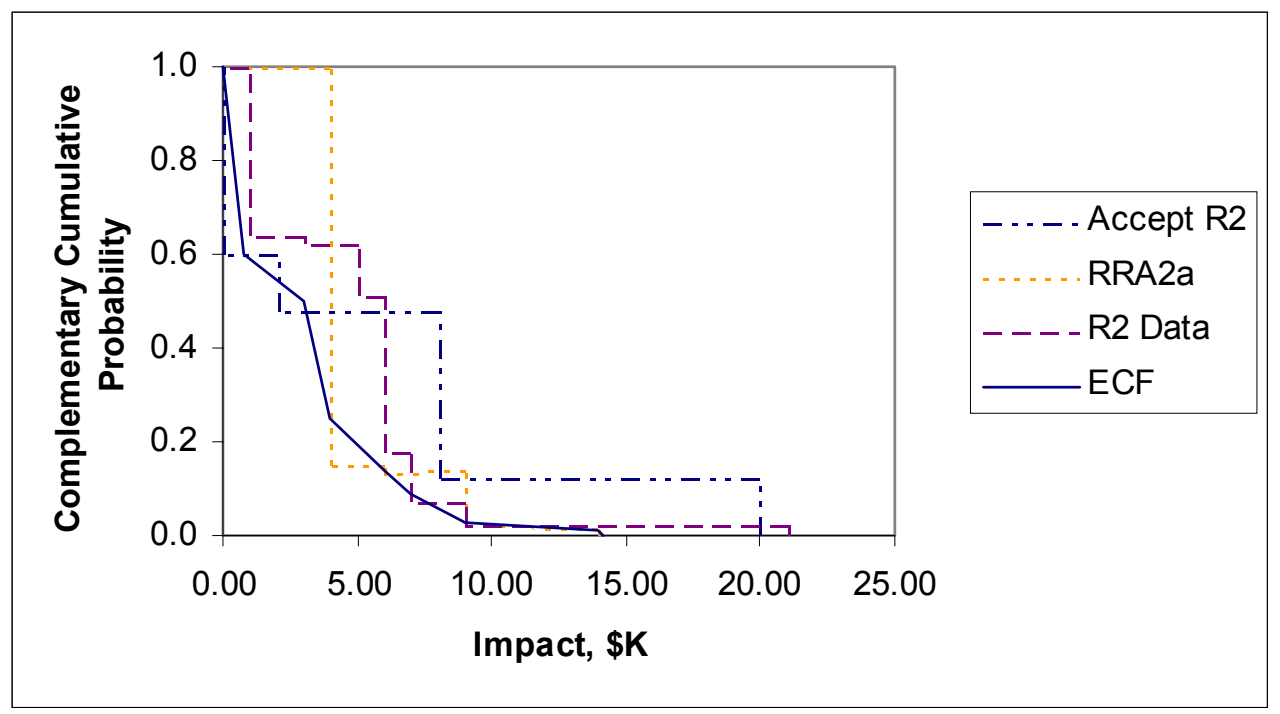

Figure 14. Cumulative risk profiles and ECF for R2 RRAs.

\section{ACKNOWLEDGMENTS}

The author would like to thank W. Bart Davis, Bill Edwards, James E. McMahon, and Xiaomin Liu (Lawrence Berkeley National Laboratory), and Barney Morais (Synergistic Applications) for detailed and insightful comments on the manuscript. This work was supported by the Director, Office of Science, Office of Basic Energy Sciences of the US Department of Energy under Contract No. DE-AC03-76SF00098.

\section{REFERENCES}

Analytica, Lumina Decision Systems, http://www.lumina.com/

P. L. Bernstein, Against the Gods: The Remarkable Story of Risk, John Wiley \& Sons, New York, 1996.

B. C. Ball and S. L. Savage, Notes on Exploration and Production Portfolio Optimization, Ball \& Savage Associates, 1999.

T. Biswas, Decision-Making Under Uncertainty, MacMillan Press, London, 1997.

J. R. Buck, Economic Risk Decisions in Engineering and Management, Iowa State University Press, Ames, 1989.

C. B. Chapman and S. C. Ward, Project Risk Management - Processes, Techniques and Insights, Wiley, Chichester, 1997.

C. B. Chapman, S.C. Ward, and J.A. Bennell, Incorporating uncertainty in competitive bidding, International Journal of Project Management 18 (2000), 337-347.

R. T. Clemen and T. Reilly, Making Hard Decisions with DecisionTools, Duxbury, Pacific Grove, 2001.

Crystal Ball, Decisioneering, http://www.decisioneering.com/.

Decision Pro 3.0, Vanguard Software Corporation, http://www.vanguardsw.com.

R. L. Dillon and M.E. Paté-Cornell, APRAM: An Advanced Programmatic Risk Analysis Method, International Journal of Technology, Policy and Management, Vol.1, No.1, (2001), 47-65. 
G. Friedman, The Intransitivity of Pairwise Comparisons Even with a Single Rational Decision Maker, Presentation at the NSF Decision-Based Workshop, Long Beach, 1999, http://dbd.eng.buffalo.edu/papers/friedman.html.

P. R. Garvey, Probability Methods for Cost Uncertainty Analysis: A Systems Engineering Perspective, Dekker, New York, 2000.

L. Gonick, Calamity Counters, Discover (May 1996), 66-67.

D. R. Graham and J. Dechoretz, The Cost-Identification \& Management System (CRIMS), AF/SMC, 1995, http://web.deskbook.osd.mil/valhtml/2/2B/2B4/2B4S08.htm.

C. N. Haas, On Modeling Correlated Random Variables in Risk Assessment, Risk Analysis 19 (1999), 1205-1214.

Y. Y. Haimes, Risk Modeling, Assessment, and Management, Wiley, New York, 1998.

Y. Y. Haimes, "Risk Management," in Handbook of Systems Engineering and Management, A. P. Sage and W. B. Rouse (Editors), Wiley \& Sons, New York, 1999.

E. M. Hall, Managing Risk: Methods for Software Systems Development, Addison-Wesley, Reading, 1998.

D. Hillson, Developing effective risk responses, Proceedings of the $30^{\text {th }}$ Annual Project Management Institute 1999 Seminars \& Symposium.

INCOSE, Systems Engineering Handbook, Release 1.0, 1998.

Insight.xla, Business Analysis Software for Microsoft Excel, Duxbury Press, Pacific Grove, 1998, http://www.stanford.edu/ savage/.

S. Kaplan, On The Method of Discrete Probability Distributions in Risk and Reliability

Calculations - Application to Seismic Risk assessment, Risk Analysis 1 (1981), 189-196.

D. Kahneman, P. Slovic, and A. Tversky, Eds., Judgement under uncertainty: Heuristics and biases, Cambridge University Press, Cambridge, 1999.

R. L. Kenney and H. Raiffa, Decisions with Multiple Objectives: Preferences and Value Tradeoffs, Wiley \& Sons, New York, 1976.

E. Kujawski, I. M. Jacobs, and A. M. Smith, An Evaluation of the Use of Signal Validation Techniques as a Defense Against Common-Cause Failures, EPRI NP-5081, Electric Power Research Institute, 1987.

E. Kujawski, SNAP Technology / Risk Opportunity Assessment, 2001, http://snap.lbl.gov/.

E. Kujawski, Why Projects Often Fail Even With High Cost-Contingencies, to be published in Systems Engineering Vol. 5, No. 2, 2002.

H. M. Markowitz, Portfolio Selection: Efficient Diversification of Investments, Yale University Press, New Haven, 1976.

H. F. Martz and R. A. Waller, Bayesian Reliability Analysis, John Wiley and Sons, New York, 1982.

N. J. McCormick, Reliability and Risk Analysis Methods and Nuclear Power Applications, Academic Press, New York, 1981.

S. R. Moran, Cost-Risk Analysis - The Devil Is In The Details, Space Systems Cost Analysis Group (SSCAG) Meeting, January 1999.

J. E. Neely III and R. de Neufville, Hybrid Real Options Valuation of Risky Product Development Projects, International Journal of Technology, Policy and Management, Vol.1, No.1, (2001), 29-46.

@Risk and PrecisionTree, Palisade Corporation, http://www.palisade.com.

S. Savage, The Flaw of Averages, San Jose Mercury News, October 8, 2000.

E. Schragenheim and H.W. Dettmer, Does Your Internal Management Meet Expectations?, CrossTalk 14 (2001) 19-25.

P. Sen and J. Yang, Multiple Criteria Decision Support in Engineering Design, SpringerVerlag, London, 1998. 
M. A. Shaikh, Project Schedule Recomputation after Risk Inclusion, Systems Engineering Vol. 1 (1998), 242-249.

Z. Shapira, Risk Taking - A Managerial Perspective, Russell Sage Foundation, New York, 1995.

W. Sharpe, Portfolio Theory and Capital markets, McGraw-Hill, New York, 2000.

U.S. Department of Defense, Joint Industry/Government Parametric Estimating Handbook, 1999.

U.S. Department of Energy, Project Management Practices, Practices 8 and 12, Draft, October 2000. 